\title{
Time to evaluate why high-volume hospitals have better surgical outcomes
}

Two studies from the Canadian Institute for Health Information (CIHI) linking hospitals that perform larger volumes of surgery to better patient outcomes are prompting another round of soul-searching over the need for centralization of certain services.

In the most comprehensive Canadian study in this area to date, CIHI researchers analyzed 30-day postoperative mortality among 180000 patients who underwent 9 types of elective surgery requiring overnight stays between April 1998 and March 2004. The information is taken from the Discharge Abstract Database and included all provinces except Quebec and Manitoba.

CIHI's 2005 Health Care in Canada report concludes that for 3 types of surgery - percutaneous transluminal coronary angioplasty; esophagectomy and Whipple surgery (pancreatico- duodenectomy) - patients fare better in Canadian hospitals with the largest volumes.

But for the other 6 types of surgery - unruptured abdominal aortic aneurysm (AAA) repair, carotid endarterectomy, colon/rectal surgery, coronary

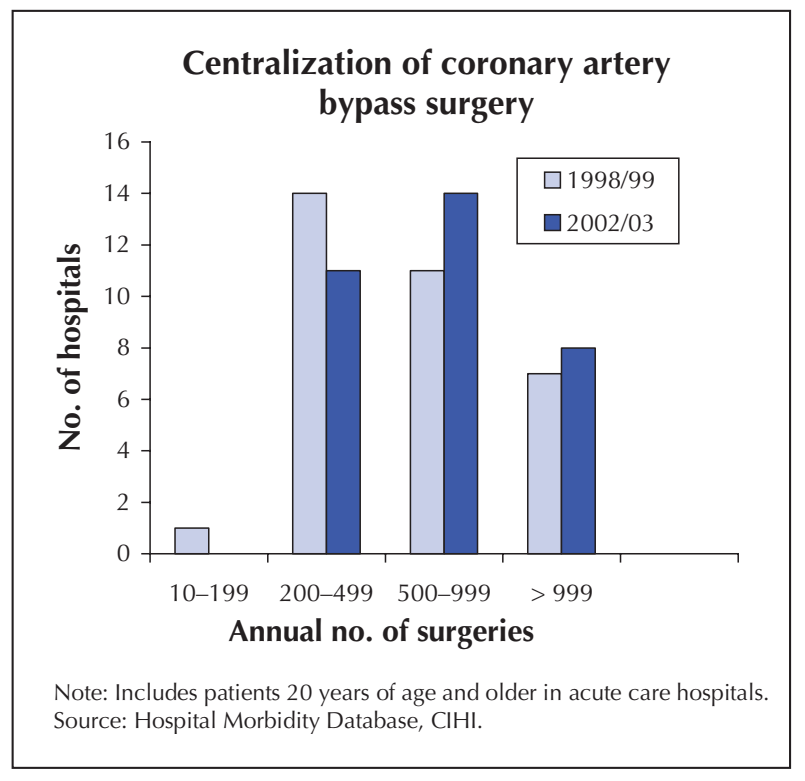

artery bypass grafting (CABG); lobectomy and pneumonectomy - the results were either inconclusive or harder to interpret.

In the 12200 surgeries for unruptured AAA repair, for example, the researchers saw no association between volume and decreased 30-day in-hospital mortality when they viewed the figures comprehensively. However, an association was seen when the highest volume quintile of hospitals was compared to the lowest volume quintile.

In a companion study, CIHI commissioned David Urbach of the Institute for Clinical Evaluative Sciences at the University of Toronto to conduct a systematic review, probing methodologies and findings of 313 analyses of hospital volumes and patient outcomes published since 1979 . Sixty-seven percent of the studies reported better results in higher-volume hospitals. Almost $80 \%$ of these studies were conducted in the US, and only $9 \%$ in Canada.

Urbach ascribes the weak results in the CIHI study compared with bigger US studies to both a lack of statistical power and a generally more favourable distribution of resources and services in Canada. For example, in the 41886 CABG surgeries that CIHI analyzed, no relationship was observed between the procedure volume and patient outcome. Jennifer Zelmer, CIHI's vice-president for research, says this may indicate that for $\mathrm{CABG}$ surgery the recent centralization has already levelled qualitative differences among Canadian hospitals (see figure).

Despite the weak results, Urbach and Zelmer agree that overall the CIHI study supports the relationship between higher volumes and improved outcomes. "The controversy is what you do about it," says Urbach.

Urbach is calling for more research probing the consequences of regional centralization of hospital services, and analyzing what medical realities actually cause higher-volume hospitals to deliver better outcomes.

Other researchers concur. After reviewing 135 volumeoutcome studies, Ethan Halm of the Mount Sinai School of Medicine in New York told CMAf that "It's important to keep in mind that the volume of surgeries conducted in a hospital is a surrogate measure of its quality. Patients should focus on a hospital's quality, where firm data is available, rather than on the number of operations it conducts."

A 2002 study of 2.5-million US cancer and cardiovascular patients, led by John Birkmeyer of New Hampshire's Dartmouth-Hitchcock Medical Center, showed a $1 \%$ difference in mortality between CABG patients at the lowest and highest volume US hospitals. "People are sick of hearing about volume as a monitor of who's best," Birkmeyer said in a phone interview. "It's time we evaluated why we've seen this."

"There are some tough decisions to be made between local availability and quality," says Graham Scott, CIHI's chairman.

Responding to the report, the Society of Rural Physicians of Canada pointed out that common procedures such as appendectomy and maternity care are done as well or better in rural hospitals closer to where patients live. The Society described the 3 types of surgery where CIHI linked volume to outcome as being "rare and highly specialized procedures."

"The good news is that we now know that even for many highly specialized surgeries, most Canadians do not need to travel to get the best possible care," says Society President Dr. Trina Larsen Soles. - Paul Webster, Toronto 